Submitted to Astrophysical Journal Letters

Preprint typeset using $\mathrm{LAT}_{\mathrm{EX}}$ style emulateapj v. 20/04/00

\title{
THE X-RAY TRANSIENT XTE J1118+480: MULTIWAVELENGTH OBSERVATIONS OF A LOW-STATE MINI-OUTBURST
}

\author{
R. I. Hynes ${ }^{1}$, C. W. Mauche ${ }^{2}$, C. A. Haswell ${ }^{3}$, C. R. Shrader ${ }^{4}$, W. Cui ${ }^{5}$, And S. Chaty ${ }^{3}$ \\ Submitted to Astrophysical Journal Letters
}

\begin{abstract}
We present multiwavelength observations of the newly discovered X-ray transient XTE J1118+480 obtained in the rising phase of the 2000 April outburst. This source is located at unusually high Galactic latitude and in a very low absorption line of sight. This made the first EUVE spectroscopy of an X-ray transient outburst possible. Together with our HST, RXTE, and UKIRT data this gives unprecedented spectral coverage. We find the source in the low hard state. The flat IR-UV spectrum appears to be a combination of optically thick disk emission and flat spectrum emission, possibly synchrotron, while at higher energies, including EUV, a typical low hard state power-law is seen. EUVE observations reveal no periodic modulation, suggesting an inclination low enough that no obscuration by the disk rim occurs. We discuss the nature of the source and this outburst and conclude that it may be more akin to mini-outbursts seen in GRO J0422+32 than to a normal X-ray transient outburst.
\end{abstract}

Subject headings: accretion, accretion disks - binaries: close - stars: individual: XTE J1118+480 - ultraviolet: stars-X-rays: stars

\section{INTRODUCTION}

Soft X-ray transients (SXTs), also known as X-ray novae, (Tanaka \& Shibazaki 1996) are low-mass X-ray binaries in which long periods of quiescence, typically decades, are punctuated by very dramatic X-ray and optical outbursts, often accompanied by radio activity. In a prototypical outburst, X-ray emission is dominated by thermal emission from the hot inner accretion disk, and optical emission is thought to be produced by reprocessing of Xrays. There are exceptions, however, and some outbursts never show disk X-ray emission (e.g., GRO J0422+32; see Nowak 1995 for summary and discussion).

XTE J1118+480 was discovered by RXTE on 2000 March 29 (Remillard et al. 2000) as a weak, slowly rising X-ray source. Analysis of earlier data revealed an outburst in 2000 January reaching a similar brightness. A power-law spectrum was seen to at least $120 \mathrm{keV}$ (Wilson \& McCollough 2000), with spectral index similar to Cyg $\mathrm{X}-1$ in the low hard state. A 13th magnitude optical counterpart was promptly identified, coincident with an object with red magnitude 18.8 in Sky Survey images (Uemura, Kato, \& Yamaoka 2000; Uemura et al. 2000). The optical spectrum was typical of X-ray novae in outburst (Garcia et al. 2000). Continued observations revealed a weak photometric modulation on a $4.1 \mathrm{hr}$ period (Cook et al. 2000). The optical brightness is surprising, as the $\mathrm{X}$-rays are so faint. It was suggested that the system might be at very high inclination, so that the X-ray source was obscured by the disk rim and only scattered X-rays are visible (Garcia et al. 2000). A radio counterpart was also discovered with flux 6.2 mJy (Pooley \& Waldram 2000).

XTE J1118 +480 has a very high Galactic latitude $(b=$ $+62^{\circ}$ ) and is close to the Lockman hole (Lockman, Jahoda, \& McCammon 1986). Consequently it has a very low interstellar absorption of $E(B-V) \lesssim 0.024$ (Garcia et al. 2000). This, together with its brightness, make XTE J1118+480 an ideal target for multiwavelength coverage. The observations described here were performed as part of an coordinated multi-wavelength campaign. A highlight of the campaign is that the low interstellar absorption allowed the first $E U V E$ spectroscopy of an X-ray transient. This campaign is still underway, so we report here only our earliest observations of 2000 April 4-18.

\section{EUVE DATA}

Extreme Ultraviolet Explorer (EUVE) observations of XTE 1118+480 took place during 2000 April 8.10-8.71, 13.32-13.93, and 16.91-19.60 UT. The deep survey (DS) photometer achieved net exposures of $0.93,0.90$, and $6.92 \mathrm{ks}$, limited by shutdowns due to high background levels. The short wavelength (SW) spectrometer was not affected by such shutdowns and achieved net exposures of 19.4, 19.8, and $81.1 \mathrm{ks}$. The bandpasses of the DS photometer and SW spectrometer are defined by a Lexan/Boron filter and extend from $\approx 70-180 \AA$ although interstellar absorption extinguishes the flux longward of about $120 \AA$. The 2 nd and 3 rd observations were offsetpointed to recover more of the short-wavelength flux.

The mean background-subtracted DS count rates for the three visits do not vary by much: 1.61, 1.51, and $1.62 \mathrm{cnts} \mathrm{s}^{-1}$. Individual light curves show some random variability (e.g., Fig. 1). To determine if this variability is correlated with orbital phase, we folded the data from the April 18 observation on the optical photometric period of $0.17082 \mathrm{~d}$ (Patterson 2000), resulting in the light

\footnotetext{
${ }^{1}$ Department of Physics and Astronomy, University of Southampton, Southampton, SO17 1BJ, UK; rih@astro.soton.ac.uk.

${ }^{2}$ Lawrence Livermore National Laboratory, L-43, 7000 East Avenue, Livermore, CA 94550, USA; mauche@cygnus.llnl.gov.

${ }^{3}$ Department of Physics and Astronomy, The Open University, Walton Hall, Milton Keynes, MK7 6AA, UK; c.a.haswell@open.ac.uk, s.chaty@open.ac.uk.

${ }^{4}$ Laboratory for High-Energy Astrophysics, NASA Goddard Space Flight Center, Greenbelt, MD 20771, USA; shrader@grossc.gsfc.nasa.gov.

${ }^{5}$ Center for Space Research, Massachusetts Institute of Technology, Cambridge, MA 02139, USA; cui@space.mit.edu.
} 
Hynes et al.

curve shown in the inset of Fig. 1. Zero phase is arbitrarily taken to occur at the start of the EUVE observation on JD 2451651.4083. There is no evidence for a modulation in the EUV flux of greater than $6 \%$ full amplitude on this period. We also performed a period search on these data but found no period significant at the $90 \%$ level, for $3 \mathrm{hr}<P<4$ day.

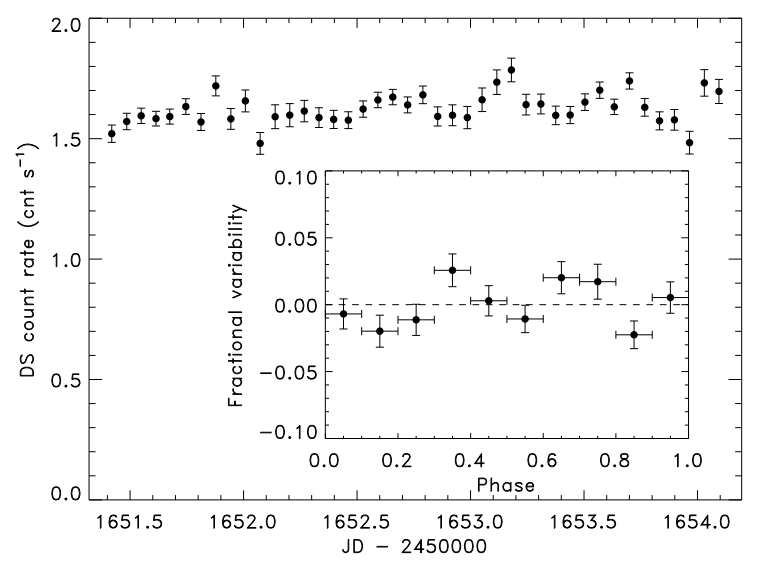

Fig. 1. EUVE/DS light curve from 2000 April 18. The error bars are the $1 \sigma$ count rate errors from the photon statistics. The inset shows the same data folded on the period of Patterson (2000), binned into 10 phase bins and converted to fractional variations about the mean.

Figure 2 shows the background-subtracted mean EUVE SW spectrum from the April 18 observation binned to $\Delta \lambda=0.5 \AA$, matching the spectral resolution, and removing the nonstatistical correlation between neighboring wavelength bins. The short-wavelength limit of the spectrum is dictated by the strong increase in the background shortward of $\sim 70 \AA$, while interstellar photoelectric absorption suppresses the flux longward of $\sim 120 \AA$. To approximately constrain the absorbing column density and flux, we fit these data with constant $F_{\lambda}$ and photoelectric absorption. For the latter, we used the EUV absorption cross sections of Rumph, Bowyer, \& Vennes (1994) for H I, He I, and He II with abundance ratios 1:0.1:0.01, typical of the diffuse interstellar medium. The resulting fit parameters and $90 \%$ confidence errors are $F_{\lambda}=\left(4.0_{-0.3}^{+0.5}\right) \times 10^{-12} \mathrm{erg} \mathrm{cm}-2 \mathrm{~s}^{-1} \AA^{-1}$ and $N_{\mathrm{H}}=$ $(8.7 \pm 0.4) \times 10^{19} \mathrm{~cm}^{-2}$, with $\chi^{2} /$ dof $=185.5 / 105=1.77$. The fit is poor because the data systematically fall below this simple model shortward of $\sim 75 \AA$, either because of a flattening of the continuum spectrum, or an absorption feature or edge. There may also be a broad emission feature at $\sim 81 \AA$. We cannot usefully constrain the slope of the EUV spectrum but assuming a wide range of intrinsic EUV spectra, $+2 \geq \alpha \geq-4$, where $F_{\nu} \propto \nu^{\alpha}$, gives a range of column densities of $(0.35-1.15) \times 10^{20} \mathrm{~cm}^{-2}$ respectively. These values are relatively low, and are fully consistent with the expected column density of the interstellar medium in the vicinity of the Lockman hole, $(0.5-1.5) \times 10^{20} \mathrm{~cm}^{-2}$ (B. Y. Welsh, private communication). There does not therefore appear to be strong absorption intrinsic to the source, although estimates from fits to the EUVE spectrum is sensitive to the assumed ionization state of the absorber.

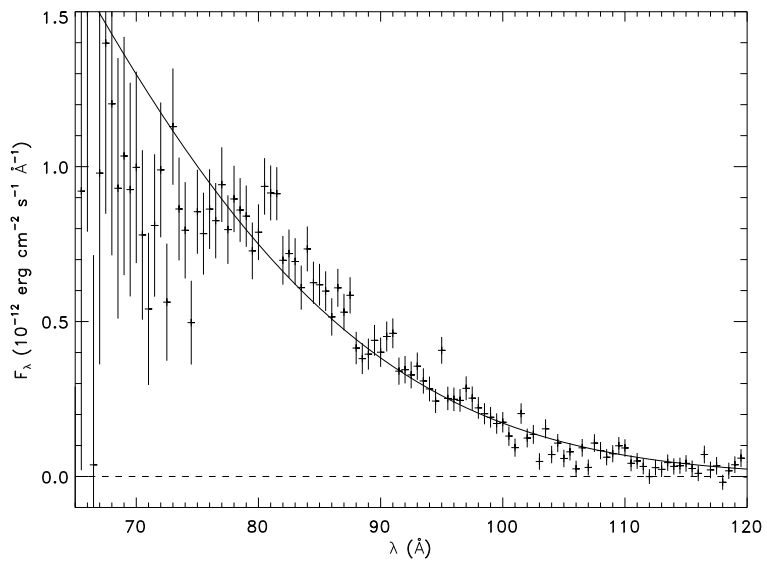

Fig. 2. Mean EUVE/SW spectrum from 2000 April 18. The vertical error bars are the $1 \sigma$ errors from the photon statistics. The fit is a constant flux, $F_{\lambda}=$ $4.0 \times 10^{-12} \mathrm{erg} \mathrm{cm}^{-2} \mathrm{~s}^{-1} \AA^{-1}$ absorbed by $N_{\mathrm{H}}=8.7 \times$ $10^{19} \mathrm{~cm}^{-2}$.

The low intrinsic absorption implied by the EUV spectrum together with the lack of orbital modulation is evidence against a high inclination explanation for the low X-ray to optical flux ratio (Garcia et al. 2000). In that case, because of the extreme sensitivity of the EUV flux to absorbing material, we would expect some modulation due to asymmetry in the disk rim and significant intrinsic absorption by material above the disk. It instead appears likely that the central disk regions are unobscured, and that the system is in an intrinsically X-ray faint state. This is also consistent with the lack of optical eclipses. This argument does, however, assume that EUV emission originates from the disk. If it actually comes from a wind, as in the cataclysmic variable OY Car (Mauche \& Raymond 2000), for example, then the system could still be at high inclination.

\section{HST AND UKIRT DATA}

Hubble Space Telescope (HST) observations were performed with the Space Telescope Imaging Spectrograph (STIS) on 2000 April 8.52-8.81 and 18.57-18.74 UT using the E140M, E230M, G430L, and G750L modes. An average calibrated spectrum for April 8 was constructed from standard HST pipeline data products. The region from $1195-1260 \AA$ was excluded as this was completely dominated by $\mathrm{N}$ V emission and Ly $\alpha$ absorption (Haswell, Hynes, \& King 2000a).

Near-infrared observations were carried out at the UKIRT $3.8 \mathrm{~m}$ telescope using UFTI $(1-2.5 \mu \mathrm{m})$ and IRCAM/TUFTI $(1-5 \mu \mathrm{m})$ on 2000 April 4 and 18 . Images were obtained in $J H K$ with UFTI and $J H K L^{\prime} M^{\prime}$ with IRCAM/TUFTI. Exposure times were $10-60 \mathrm{~s}$ and the conditions were photometric. The images were processed by removal of the dark current, flat-fielding, and sky subtraction. The magnitudes acquired on April 4.2 UT with UFTI are: $J=12.12 \pm 0.02, H=11.75 \pm 0.02$, and $K=11.06 \pm 0.02$ (Chaty et al. 2000). The magnitudes acquired on April 18.5 UT with IRCAM/TUFTI are: $J=11.92 \pm 0.07, H=11.43 \pm 0.06, K=11.05 \pm 0.08$, $L^{\prime}=9.71 \pm 0.14$, and $M^{\prime}=9.38 \pm 0.42$. The source brightened between these two dates, which bracket the dates of 
HST observations described above.

From UV to IR wavelengths $(\sim 1000-50000 \AA)$ the spectrum is flat in $F_{\nu}$ to within $10 \%$, although a weak Balmer jump in absorption of $\sim 7 \%$ is present. This likely indicates that some optically thick disk emission is present. The underlying flat continuum, however, does not resemble a disk spectrum, as is discussed in Section 5 .

\section{RXTE DATA}

We observed XTEJ1118+480 with the Rossi X-ray Timing Explorer (RXTE) Proportional Counter Array (PCA) and High-Energy Timing Experiment (HEXTE) at several epochs selected to coincide with the $H S T$ visits. This included 2000 April 8.55-8.58 when a total exposure of about $4 \mathrm{ks}$ was obtained. Subsequent discussion refers to that observation.

For the PCA, we used the "standard mode" data (128 spectral channels, $16 \mathrm{~s}$ accumulations), selecting subintervals when the number of detectors on remained constant (about $75 \%$ of the total). The current PCA background model and/or the instrument responses are unreliable above about $25 \mathrm{keV}$, so we did not use those channels for our model fitting. There was sufficient PCA-HEXTE overlap that this was not a serious limitation. The lowest several energy channels are also known to be problematic so were discarded. The total flux on the $2-10 \mathrm{keV}$ band was about $1.0 \times 10^{-9}$ ergs cm ${ }^{-2} \mathrm{~s}^{-1}$, i.e. about $40 \mathrm{mCrab}$. For HEXTE, the exposure time was rather marginal for detailed spectral modeling given the source intensity relative to the background, but the source seems to be solidly detected to $\sim 80 \mathrm{keV}$, and perhaps above $100 \mathrm{keV}$.

The data sets were then simultaneously deconvolved to derive a best estimate of the incident photon flux. Given the known cross-calibration discrepancies between the two instruments, we allowed the two normalization terms to vary independently. The spectrum is hard, with a photon power-law index of about $1.8 \pm 0.1$. A thermal Comptonization model (Sunyaev \& Titarchuk 1980) with $\tau=3.2$ and $T_{\mathrm{e}}=33 \mathrm{keV}$, also provided an acceptable fit. In both cases, there was a distinct positive residual corresponding to the $6.4 \mathrm{keV}$ Fe $\mathrm{K}$ resonance, thus a Gaussian line profile was included to refine our overall fit. Given the low hydrogen column density, and our lack of low-energy coverage, the effect of interstellar absorption on the X-ray spectrum is negligible. There was no evidence (in terms of statistical improvement to our fits) for a soft-excess component.

\section{THE SPECTRAL ENERGY DISTRIBUTION}

Figure 3 shows the spectral energy distribution (SED) inferred from radio to hard X-ray data. Radio data obtained with the VLA and Ryle telescope between 2000 April 2.74-3.13 were reported by Dhawan et al. (2000). Between then and April 18, the radio was no more than $30 \%$ brighter, i.e. $\Delta \log F_{\nu} \lesssim 0.1$ (G. Pooley, private communication). UKIRT data were obtained on April 4 and 18 and are consistent to within $\Delta \log F_{\nu} \sim 0.15$. The other data were obtained simultaneously during April 8. Calibration errors in the HST and RXTE data are estimated at $\Delta \log F_{\nu} \lesssim 0.1$. The EUVE uncertainty is dominated by the uncertain absorption as shown.

These data have been corrected for interstellar absorption where necessary. Assuming the column density inferred above to be interstellar, and adopting an average gas-dust ratio (Bohlin, Savage \& Drake 1978) and extinction curve (Seaton 1979), we corrected the IR-UV data for reddening with $E(B-V)=0.013$. This makes only a small difference at these wavelengths as the value is so low. The assumed absorption is important at EUV wavelengths so for the $E U V E$ data we plot three solutions with $N_{\mathrm{H}}=0.35,0.75$, and $1.15 \times 10^{20} \mathrm{~cm}^{-2}$ to illustrate how the flux and spectral index vary with assumed absorption.

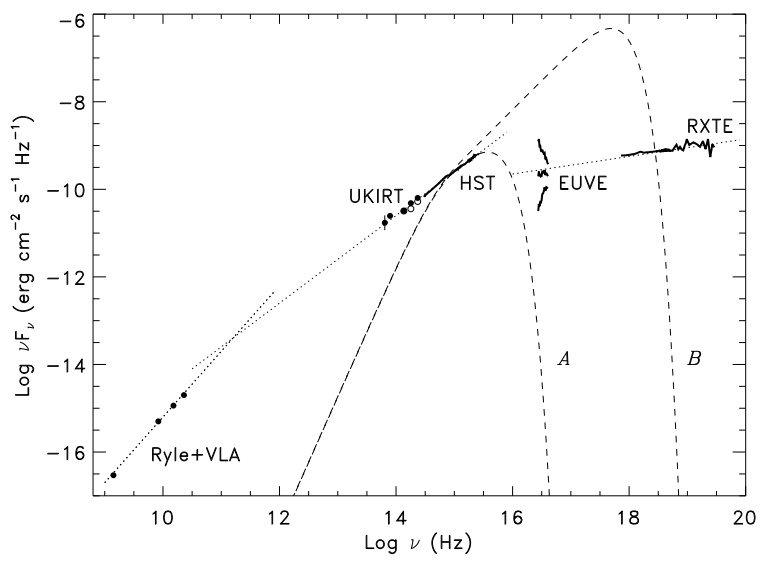

Fig. 3. Spectral energy distribution from radio to hard X-ray frequencies on 2000 April 8 (HST, EUVE, RXTE, April 2-3 (radio), and April 4, 18 (UKIRT). EUVE data are shown corrected for three different amounts of absorption. Dashed lines are two steady-state disk models with differing inner radii. Dotted lines are characteristic powerlaws $\left(F_{\nu} \propto \nu^{\alpha}\right.$; from left to right, $\left.\alpha=+0.5,0.0,-0.8\right)$. See text for details.

The presence of a Balmer jump in the optical data suggests that an optically thick disk makes a significant contribution there. Fig. 3 shows model SEDs calculated for a steady-state viscously heated disk (Shakura \& Sunyaev 1973). These are intended to be schematic rather than detailed fits. The low energy cut-off is fixed by an outer temperature of $8000 \mathrm{~K}$, chosen to be consistent with the hot phase of the disk instability model. The high energy cut-off then depends on the inner radius: $\sim 3 \mathrm{R}_{\mathrm{s}}$ and $\sim 2000 R_{\mathrm{S}}$ for models $\mathrm{A}$ and $\mathrm{B}$ respectively. Model A is representative of a high state SED, with the disk extending to the last stable orbit. This is clearly ruled out by the EUV and X-ray data as a direct consequence of the low X-ray to optical flux ratio. The uncertainty in the system parameters, including the possibility that the central object is a neutron star, cannot resolve this. Model B is more like the low hard state scenario proposed by Esin et al. (1997) in which the center of the disk (out to $10^{3}-$ $10^{4} \mathrm{R}_{\mathrm{S}}$ ) is evaporated into an advective flow. This model resolves the EUV/X-ray problem and reproduces the UV slope fairly well. The EUV to hard X-ray range can be approximately fitted by a single power-law, $F_{\nu} \propto \nu^{-0.8}$, typical of the low hard state, and so would not be disk emission. This spectral region does resemble the low hard state SEDs presented by Esin et al. (1997), as does the Xray to optical flux ratio. Alternative explanations of this low flux ratio include material leaving the system in an outflow (e.g., Blandford \& Begelman 1997), or accumulating at larger radii in a non-steady state disk; either option 
Hynes et al.

will reduce the central accretion rate as required.

Disk models provide a very poor fit in the IR, as a disk spectrum should steepen to a Rayleigh-Jeans tail well before the $M^{\prime}$ band is reached (unless the edge of the disk is at $T \lesssim 1000 \mathrm{~K}$ ). It thus appears that another source of near-IR flux is present. This may be related to the radio emission, as this lies close to an extrapolation of the IR-UV power-law. The very flat IR-UV part of the SED $\left(F_{\nu} \sim\right.$ constant $)$ could then very naturally be interpreted as a mixture of an optically thick disk spectrum and flatspectrum emission, possibly synchrotron, such as is seen at radio-mm wavelengths in Cyg X-1 and other black hole candidates (Fender et al. 2000). The radio emission has a steeper, inverted spectrum $\left(F_{\nu} \propto \nu^{0.5}\right)$, likely optically thick synchrotron emission.

\section{DISCUSSION}

Consideration of the spectral energy distribution suggests that during the period 2000 April 4-18 the source was in the low hard state. Power density spectra at X-ray, UV, and optical wavelengths support this, showing bandlimited noise and QPOs typical of this state (Revnivtsev, Sunyaev, \& Borozdin 2000; Haswell et al. 2000b). This in itself is not particularly striking: GRO J0422+32, for example, spent its entire 1992-1993 main outburst in the low hard state. What is striking is the low X-ray to optical flux ratio: it is a very low low state. This is not typical of a major SXT outburst. There are, however, similarities in this behavior to mini-outbursts in GRO J0422+32 (CastroTirado, Ortiz, \& Gallego 1997; Shrader et al. 1997). Like the outbursts of XTEJ1118+480, these outbursts were characterized by a very low X-ray to optical flux ratio and low hard state behavior. The optical outbursts have comparable length and brightness; in GRO J0422+32 the mini-outburst duration is $\sim 60 \mathrm{~d}$ with $\sim 120 \mathrm{~d}$ recurrence time. In XTE J1118+480 the first outburst lasted $\sim 40 \mathrm{~d}$, with the second outburst beginning $\sim 60 \mathrm{~d}$ after the start of the first. In both cases, the peak optical brightness is $\sim 6$ mag above quiescence, with the interoutburst brightness about $3.5 \mathrm{mag}$ above quiescence in XTE J1118+480 and $1-3 \mathrm{mag}$ above quiescence in GRO J0422+32. It thus appears likely that the outburst of XTE J1118+480 is of the same kind as the mini-outbursts in GRO J0422+32, suggesting that this phenomenon is not just an aftereffect of a main outburst, but can occur in isolation.

The nature of the compact object remains uncertain. The spectral energy distribution is equivocal, as both black hole and neutron star systems can show extended powerlaws in the low hard state, with similar photon indices (Barret et al. 2000 and references therein). A distinguishing neutron star feature may be a very soft excess, $T \sim 0.5 \mathrm{keV}$, associated with the heated neutron star surface or boundary layer, however, our data clearly cannot address this. We note that Revnivtsev et al. (2000) have speculated that the compact object is a black hole based on the non-detection of high frequency variability, which is considered a signature of a neutron star.

The EUVE observations were made possible by a generous grant of Director's Discretionary Time by EUVE Project Manager R. Malina, the efforts of EUVE Science Planner M. Eckert, the staff of the EUVE Science Operations Center at CEA, and the Flight Operations Team at Goddard Space Flight Center. This work includes observations with the NASA/ESA Hubble Space Telescope, obtained at the Space Telescope Science Institute, operated by the Association of Universities for Research in Astronomy, Inc. under NASA contract No. NAS5-26555. We would like to thank the $H S T$ and RXTE support staff for ongoing efficient support. UKIRT is operated by the Joint Astronomy Centre on behalf of the U.K. Particle Physics and Astronomy Research Council. We thank the JAC, and in particular J. K. Davies, for their open policy and efficiency and G. P. Smith and I. Smail for their assistance with the April 4 observation. RIH, CAH, and SC acknowledge support from grant F/00-180/A from the Leverhulme Trust. CWM's contribution was performed under the auspices of the U.S. Department of Energy by University of California Lawrence Livermore National Laboratory under contract No. W-7405-Eng-48. WC acknowledges NASA LTSA grant NAG5-7990.

\section{REFERENCES}

Barret, D., Olive, J. F., Boirin, L., Done, C., Skinner, G. K., \& Grindlay, J. E., 2000, ApJ, 533, 329

Blandford, R. D., \& Begelman, M. C., 1999, MNRAS, 303, L1

Bohlin, R. C., Savage, B. D., \& Drake, J. F., 1978, ApJ, 224, 132

Castro-Tirado, A. J., Ortiz, J. L., \& Gallego, J., 1997, A\&A, 322, 507

Chaty, S., Haswell, C. A., Smith, G. P., Smail, I., \& Hynes, R. I., 2000, IAU Circ. 7394

Cook, L., Patterson, J., Buczynski, D., \& Fried, R., 2000, IAU Circ. 7397

Dhawan, V., Pooley, G. G., Ogley, R. N., \& Mirabel, I. F., 2000, IAU Circ. 7395

Esin, A. A., McClintock, J. E., \& Narayan, R., 1997, ApJ, 489, 865

Garcia, M., Brown, W., Pahre, M., McClintock, J., Callanan, P., \& Garnavich, P., 2000, IAU Circ. 7392

Fender, R. P., Pooley, G. G., Durouchoux, P., Tilanus, R. P. J., \& Brocksopp, C., 2000, MNRAS, 312, 853

Haswell, C. A., Hynes, R. I., \& King, A. R., 2000a, IAU Circ. 7407

Haswell, C. A., Skillman, D., Patterson, J., Hynes, R. I., \& Cui, W., 2000b, IAU Circ., submitted
Lockman, F.J., Jahoda, K., \& McCammon, D., 1986, ApJ, 302, 432

Mauche, C. W. \& Raymond, J. C., 2000, ApJ, 541, in press (astro-ph/0004414)

Nowak, M. A., 1995, PASP, 107, 1207

Patterson, J. 2000, IAU Circ., 7412

Pooley, G. G., \& Waldram, E. M., 2000, IAU Circ., 7390

Remillard, R., Morgan, E., Smith, D., \& Smith, E., 2000, IAU Circ. 7389

Revnivtsev, M., Sunyaev, R., \& Borozdin, K., 2000, A\&A, submitted (astro-ph/0005212)

Rumph, T., Bowyer, S., \& Vennes, S. 1994, AJ, 107, 2108

Seaton, M. J., 1979, MNRAS, 187, 73P

Shakura N. I. \& Sunyaev R. A., 1973, A\&A, 24, 337

Shrader, C. R., Wagner, R. M., Charles, P. A., Harlaftis, E. T., \& Naylor, T., 1997, ApJ, 487, 858

Sunyaev, R. A. \& Titarchuk, L. G., 1980, A\&A, 86, 121

Tanaka, Y., Shibazaki, N., 1996, ARA\&A, 34, 607

Uemura, M., Kato, T., \& Yamaoka, H., 2000, IAU Circ. 7390

Uemura, M. et al., 2000, PASJ, submitted (astro-ph/0004245)

Wilson, C. A. \& McCollough, M. L., 2000, IAU Circ. 7390 